\title{
Application of Reference Levels in the Existing Exposure Situations
}

-Towards Stepwise Reduction of Radiation Dose Depending on Situations of Contamination-

\author{
Dependi \\ Central Research Institute of Electric Power Industry \\ Haruyuki Ogino, Nobuyuki Hamada and Daisuke Sugiyama
}

\begin{abstract}
Optimizing protection against radiation on the reference levels is important to restore the contaminated areas after an accident. Various steps, which are decided based on the contamination status, have to be performed to achieve normality by considering the balance between the health risks of radiation and the societal and economic effects. This study discusses the idea of reference levels that has been introduced by the International Commission on Radiological Protection (ICRP). Further, we will discuss three measures that have been introduced after the Fukushima Daiichi Nuclear Accident, namely the decontamination screening levels, provisional regulation values and new standard values of food, and target values for the management of radioactive contaminated substances, and consider the application of intermediate reference levels in phases.
\end{abstract}

\section{Concept of Protection against Low-Dose Radiation}

After the Fukushima Daiichi Nuclear Power Plant Accident, there were concerns and fears related to the effect of low-dose radiation on health and the concept of protection against radiation such as "why were the protection standards different after an accident?" and "given the fact that new standards were introduced for food, were the old standards dangerous?"

There is a considerable amount of scientific knowledge related to the human health effect of high-dose radiation. However, debates are observed to persist at an international level over the human health effect of low-dose radiation below $100 \mathrm{mSv}$. This is because of the difficulty in distinguishing the effect of radiation from the effect of other factors. There is some knowledge with regard to the range of effects of low-dose radiation. However, the impression that enough scientific knowledge is not known has led some people to consider the unknown to be dangerous, further increasing the fear among the public.

The International Commission on Radiological Protection (hereafter, the ICRP) maintains that, from the viewpoint of protection against radiation, even low-dose radiation is assumed to exhibit effects according to the dose and that actions should be taken to reduce the exposure

DOI : 10.15669/fukushimainsights.Vol.1.256

(C) 2021 Atomic Energy Society of Japan. All rights reserved.

Originally published in Journal of the Atomic Energy Society of Japan (ISSN 1882-2606), Vol. 55, No. 2, p. 106-110 (2013)

in Japanese. (Japanese version accepted: December 10, 2012) 
as low as reasonably achievable, economic and societal factors being taken into account. Due to this, there are different threshold values that separate "safe" and "dangerous" from tissue reactions (deterministic effects) such as skin erythema; further, there is no threshold for the probabilistic effects such as cancer. Therefore, reference levels have been introduced as restrictions to serve as countermeasures to reduce exposure.

Strict application of the standards (dose limit) after an accident, which are set for normal conditions, can cause massive evacuations and confusion during daily activities, which could affect the society and the economy. Therefore, ICRP introduced the basic concept of protection against radiation, which ensures balance with the health impact of radiation by temporarily increasing the allowed dose from the normal value to a "reference level" with an annual effective dose of $100 \mathrm{mSv}$ or less through which normality can be achieved in phases.

On July 19, 2011, the Nuclear Safety Commission (NSC), Japan decided that it was appropriate to apply the concept of reference levels that was introduced by ICRP in $2007^{1)}$ to serve as radiation protection as part of restoration. In future, it will be important to deepen our knowledge with regard to the reference levels and to reduce the exposure to restore the contaminated areas.

This study will examine the advice given by ICRP in $2007^{1)}$ with regard to reexamining the principles and rules of protection against radiation and will discuss the concept of reference levels. This study will also discuss three examples of measures that are introduced after the Fukushima Daiichi Nuclear Power Plant Accident, including the decontamination screening levels, provisional regulation values and new standard values of food, and target values for radioactive contaminated substances; further, this study will consider the application of intermediate reference levels in phases. In addition, you may refer to the lecture series of this journal that was released in 2010, "ICRP's new recommendation - new concepts and standards for protection against radiation" (8 installments), for the ICRP 2007 Recommendations ${ }^{1)}$.

\section{Principles and Rules of Protection against Radiation}

\section{Approach for the Radiation Sources}

Before the ICRP 2007 Recommendations ${ }^{1)}$, the ICRP 1990 Recommendations ${ }^{2)}$ stated that the contribution of a radiation source (explained in the subsequent chapter) is not related to that of other sources when an individual dose is sufficiently lower than the threshold value that is observed in case of deterministic effects. An individual can be exposed to multiple sources; however, to ensure protection against radiation, it should be possible to independently treat each source or source group, which will ensure that all the individuals who are potentially exposed by sources or source groups are considered. This procedure can be referred to as the "source-related approach," and the ICRP 2007 Recommendations ${ }^{1)}$ emphasized that this approach was the most important in terms of protection. Even though the source-related approach may not provide sufficient protection in case of multiple radiation sources, we observe that there is a dominant source in majority of the situations; therefore, an appropriate selection of the reference level will guarantee an appropriate level of protection.

\section{Definition of the Radiation Source}

While using the source-related approach, it is necessary to define the radiation source. In the ICRP 2007 Recommendations ${ }^{1)}$, examples of radiation sources included single physical 
sources (e.g., radioactive material and X-ray devices), facilities (e.g., hospitals, nuclear power plants), and physical sources with methods or similar characteristics (nuclear medical methods, backgrounds, or environmental radiation). If a facility emitted radioactive materials into the environment, the entire facility was considered to be a single radiation source. If radioactive materials were already scattered in the environment, partial collection of radioactive materials would be considered to be a radiation source. In addition, the definition of a radiation source was generally assumed to be associated with the selection of an appropriate protection strategy; therefore, a radiation source can be defined during the process of establishing countermeasures.

\section{Exposure Situations}

The ICRP 1990 Recommendations ${ }^{2)}$ adopted protection methods based on practice (human activity that increased individual exposure and the number of people who were exposed) and intervention (human activity for reducing the overall exposure). This concept was further developed in the ICRP 2007 Recommendations ${ }^{1)}$, and protection methods were adopted for three exposure situations (planned exposure situations, emergency exposure situations, and existing exposure situations). The definition of each exposure situation is given below.

A planned exposure situation involves intentional introduction and operation of radiation sources. This can cause expected exposure (normal exposure) as well as unexpected exposure (potential exposure).

An emergency exposure situation requires emergency countermeasures to avoid or minimize the effect of situations that are caused while operating planned activities; these situations may range from malicious intent to other unexpected situations.

An existing exposure situation is a situation that already exists when a decision on control has to be taken. A long-term exposure after the occurrence of emergencies is included in this situation.

\section{Principles of Protection Against Radiation}

The source-related principles, which are applied to all the exposure situations, include the justification and optimization of protection. The individual-related principles, which are applied in case of planned exposure situations, include the dose limit. The definition of each principle is given below.

Justification: Any decision that affects the radiation exposure situation will ensure that the advantage is greater than the disadvantage. By adding new radiation sources, reducing the existing exposure, and reducing the risk of latent exposure, individual or societal advantages that cancel out the damage caused by such risks will be observed.

Optimization of protection: The possibility of exposure, number of exposed individuals, and individual dose for such individuals should be kept as low as reasonably achievable, economic, and societal factors being taken into account.

The level of protection should be optimal under the general conditions; further, the extent to which the advantage exceeds the disadvantage must be maximized. To avoid the occurrence of unfair results using the optimization method, the dose constraint, the risk constraint, and the reference level should be set to restrict the doses from and the risks of specific radiation sources.

Dose restrictions: In planned exposure situations, except for medical-related exposures in case of patients, the total individual dose from any regulated source must not exceed the dose limit that is specified by ICRP. 


\section{Dose Limit and Reference Level}

To provide protection against radiation for the general public in planned exposure situations, individual exposure has been managed to ensure that the effective dose does not exceed $1 \mathrm{mSv}$ per year, except the natural radiation and medical exposures. To ensure that the general public is protected against the radiation under existing exposure situations, it is recommended that the reference level should be set to approximately 1-20 mSv per year; further, optimized protection should be implemented. Thus, we can infer that $1 \mathrm{mSv}$ per year can be considered to be the limit of individual exposure in planned exposure situations as well as the lower bound of the source-related reference level in the existing exposure situations.

What level of risk does the exposure to $1 \mathrm{mSv}$ per year exhibit? According to the ICRP 1990 Recommendations $^{2)}$, there were at least two objectives behind setting the dose limit at $1 \mathrm{mSv}$ per year. The first was the decision from the radiation risk estimation model that was based on the epidemiological studies that were conducted with the help of the survivors from the nuclear attacks at Hiroshima and Nagasaki. Using the multiplicative risk model (assuming that an increase in the ratio of the mortality rate due to radiation to the natural mortality rate continues for a lifetime) and by assuming that the dose and dose-rate effectiveness factor (determined using a decision for which the biological effect per unit dose at low doses and a low dose-rate is lower than that in high doses and a high dose-rate) is 2,75 years of exposure at $1 \mathrm{mSv}$ per year would result in the death of 95 out of 1 million people (not exceeding $10^{-4}$ ). The second objective was that the decision was made based on the range of variation in the natural background radiation. Except in the case of exposure to radon, which is easy to change, the dose from the natural background radiation is approximately $1 \mathrm{mSv}$ per year, and elevated areas exhibit doses that are more than twice this value.

Thus, the value of $1 \mathrm{mSv}$ per year has been chosen as the public dose limit in case of planned exposure situations after considering the radiation risk estimation models and the natural background radiation levels from various angles. The public dose is managed so that it is within the dose limit that is set by certain considerations; further, it is important to understand that the dose limit is not set as an indicator for safety and danger.

Once a nuclear accident or a radiation emergency occurs, the environment in which the radiation sources exist, which are assumed to ensure radiation risk management, changes. The characteristics of protection also change, and matters ranging from the deterministic effects on human health to effects on quality of life will be handled. Such situations that require emergency measures or emergency exposure situations lead to decisions that differ from regular decisions in which the dose limit is not applied; thus, the concept of a reference level emerges.

As time passes after the occurrence of a nuclear accident or a radiation emergency, the radiation measurements are performed by various bodies; further, the knowledge of the environment in which the radiation source exists is collected. Later, an existing exposure situation is observed in which long-term coexistence of manageable radiation sources and humans are taken into consideration. In an existing exposure situation, the dose limits are not applied; further, the objective is to ensure the optimization of protection against radiation based on the reference levels. To implement optimization, which is to keep the exposure as low as reasonably achievable, economic and societal factors being taken into account, the lessons learned from the Chernobyl Accident ${ }^{3)}$ indicate the importance of making decisions based on the inputs of the residents and the autonomous activities in individual living environments. 


\section{Discussion on the Application of Reference Levels}

Implementing the optimization principle is a source-related process, and an optimal protection strategy is selected for the given prevailing circumstances; further, the difference between advantage and disadvantage should be maximized. ICRP recommends that the reference level in the existing exposure situations should be $1-20 \mathrm{mSv}$ per year ${ }^{1)}$. The recommendations also indicate that the long-term objective of an existing exposure situation is to restore the dose level back to normal or equivalent to the normal level; as such, the reference level should be in the lower part of the $1-20 \mathrm{mSv}$ per year range ${ }^{3)}$. ICRP also recommends that the governmental regulatory agencies should consider the given prevailing circumstances, use the timing of restoration planning, and apply an intermediate reference level to improve the situation in phases. Based on experience, the representative value to restrict the optimization process in the long-term period after an accident is $1 \mathrm{mSv}$ per year. Here, $1 \mathrm{mSv}$ per year should be considered as the lower bound of the reference level for radiation sources in existing exposure situations in such a context.

The optimization process is to be applied to situations in which the protection strategy is justified; further, it requires judgments, which demand transparency. To make decisions based on sufficient explanations, transparency requires that all the related information should also be provided to stakeholders and that the traceability of the decision-making process should be appropriately documented.

The following is a discussion on the three examples of measures that are introduced after the Fukushima Daiichi Nuclear Power Plant Accident, such as the decontamination screening levels, provisional regulation values and new standard values of food, and target values for management of radioactive contaminated substances; further, we consider the application of intermediate reference levels in phases.

\section{Decontamination Screening Levels}

In case of regular radiation management, humans are observed to vacate the managed areas. When materials are to be taken out, the density of radioactive materials on the surface of the body or the materials must be lower than 1/10th of the surface density limit (i.e., $4 \mathrm{~Bq} / \mathrm{cm}^{2}$ for alpha-ray-emitting radionuclides and $40 \mathrm{~Bq} / \mathrm{cm}^{2}$ for non-alpha-ray-emitting radionuclides). At a site under regular radiation management, when significant contamination is detected, it is a general practice to decontaminate a reasonable area near the location and to eliminate the concerned material even while conforming to the standards that are defined in regulations such as the Radiation Hazard Protection Act.

However, the accident simultaneously contaminated humans and materials over a wide range of areas. Furthermore, decontaminating every person was impossible due to water shortages and blackouts after the disaster in Japan; additionally, the low temperatures made it difficult to remove clothes. In such a situation, it was not realistic to strictly apply the regular standards. It became necessary to use a scientific standard to classify individuals and materials based on their priorities for decontamination. This requires screening (sorting). Based on the perspective of emergency medical exposure, the screening level in Fukushima was set to $40 \mathrm{~Bq} / \mathrm{cm}^{2}$ (equivalent to $13,000 \mathrm{cpm}$ (counts per minute) measured by a GM survey meter, a general radiation meter). Given the aforementioned situation, the screening level was set to $100,000 \mathrm{cpm}$, which was the maximum reading of a GM survey meter, after March 14, 2011. $\mathrm{Cpm}$ is the number of measurements of the radiation meter per minute.

The authors assessed the whole-body effective dose and local skin absorbed dose ${ }^{4)}$ by 
assuming a case in which surface contamination of $100,000 \mathrm{cpm}$ was observed on a material and in which a similar amount of contamination was directly deposited on the skin. The results exhibited that the whole-body effective dose from the material was below $1 \mathrm{mSv}$ per year, which was the lower limit of source-related reference level, and that the local skin-absorbed dose was also lower than $1 \mathrm{mGy}$ per hour. By considering a realistic exposure time, the skin was observed to be protected from deterministic effects such as hair loss and ulcers (threshold is of the Gy order) ${ }^{4)}$.

On August 29, 2011, the NSC recommended that the screening levels should be reduced in phases to prevent further expansion of contamination by radioactive materials outside the evacuation areas (restricted areas). Based on this advice, the director-general of the Local Nuclear Emergency Response Headquarters set 13,000 cpm as the new screening level after September 16, 2011. In future, it will be important to consider the progress of the monitoring results and to flux in and out of evacuation areas (restricted area), to consider the connectivity with the regular standards, and to continue the efforts to reduce the screening levels in phases.

\section{Provisional Regulation Values and New Standard Values for Food}

The regulation of food contaminated by radioactive materials is important to reduce internal exposure. The NSC had set indicators before the accident so that disaster countermeasure offices will use them to initiate discussions with regard to whether food regulation was necessary; however, there were no regulation values in case of food regulation. Based on the indicator values, the Ministry of Health, Labour, and Welfare set the provisional regulation value for radioactive material in food on March 17, 2011; further, the shipping and intake of food, which violated the provisional regulation values, were regulated.

The authors sorted the reasons for setting food regulations and provisional regulation values that were implemented within the initial three months after the accident, extracted issues in food regulation, and proposed an improvement plan $^{5,6)}$. We also organized the food regulations that were implemented within the first year after the accident ${ }^{7)}$. We highlighted the issues in food regulation, including a lack of comprehensive indicator values for radioiodine in case of a variety of food, and the fact that the original objective for setting the indicator values was compromised when the provisional regulation values for seafood were added and when the provisional regulation values for drinking water and dairy were altered. Furthermore, we exhibited that social confusion had occurred since a similar indicator value was set (equivalent to an intervention dose level of $5 \mathrm{mSv}$ ) without distinguishing the emergency exposure situation and the existing exposure situation after the accident.

To improve upon such issues, the authors have proposed to implement the reference levels in phases ${ }^{5,6}$. This idea uses the difference in exposure based on the distance from the accident site, considers the effectiveness of the regulation values, and categorizes the periods between the emergency exposure situation and existing exposure situation during an early phase (phase 1), an interim phase (phase 2), and a later phase (phase 3) and reduces public exposure in phases by setting the reference levels for each phase. This concept also involves setting operational reference levels that are equivalent to the regulation values for each phase. The operational reference levels are set to be applied to all the food, radioactive isotopes, and age groups in such a manner that they do not extend beyond the reference levels in each phase. With regard to selecting the reference levels for each phase, we consider it appropriate to use $10 \mathrm{mSv}$ per year, which is 1/10th of the upper bound of the reference level during an emergency exposure situation (100 mSv per year), for an early phase, $1 \mathrm{mSv}$ per year, which is the 
lower bound of the reference level during an existing exposure situation, for the later phase, and $5 \mathrm{mSv}$ per year, which is somewhere between 10 and $1 \mathrm{mSv}$ per year, for the interim phase $^{5,6)}$.

On April 1, 2012, the Ministry of Health, Labour, and Welfare decided to further ensure food safety and set new standard values for food to replace the provisional regulation values and to prepare for long-term situations. The additional dose from contaminated food was reduced from 5 to $1 \mathrm{mSv}$ per year, and a standard value was established for radioactive cesium. This action can be understood to consider the contamination of food as one radiation source group using $1 \mathrm{mSv}$ per year, which is the lower bound of the reference value for the existing exposure situations.

\section{Target Values for Management of Radioactive Contaminated Substances}

After the accident, a significant amount of substances contaminated with released radionuclides, such as debris, waste matter, sludge, incinerated ash, and soil removed due to decontamination, among others, hereafter referred as contaminated substances, were generated over a wide area. It was decided that safety with regard to managing the contaminated substances should be based on the ideas provided in "ideas on immediate handling of by-products containing radioactive materials from drinking water and sewage treatment facilities" by the Nuclear Emergency Response Headquarters. This idea holds that the dose for the surrounding residents after the management period of the disposal facility should be lower than $0.01 \mathrm{mSv}$ per year under a scientifically probable scenario (likely scenario) and lower than $0.3 \mathrm{mSv}$ per year in case of variations of the likely scenario (less-likely scenarios). To ensure that the dose for the surrounding residents during the operational phase or for the operator is lower than $1 \mathrm{mSv}$ per year, the disposal of contaminated substances with a radioactive cesium concentration lower than $8,000 \mathrm{~Bq} / \mathrm{kg}$ is allowed, and contaminated substances with a radioactive cesium concentration lower than $100,000 \mathrm{~Bq} / \mathrm{kg}$ can be temporarily stored at facilities equivalent to a controlled landfill waste disposal facility for municipal wastes that do not contain radioactive materials in normal situation.

The aforementioned target values are based on the guidelines ${ }^{8)}$ for the management of radioactive waste in planned exposure situations. In addition, the value of $1 \mathrm{mSv}$ per year refers to individual dose limits in planned exposure situations. This can be interpreted as consideration that suitable management of contaminated substances was necessary in remote areas from the Fukushima Daiichi Nuclear Power Plant with low radioactive contamination.

However, while applying the aforementioned target values to environmental remediation (such as decontamination) in areas with environmental radiation of a few $\mathrm{mSv}$ to few tens of $\mathrm{mSv}$ per year due to soil contamination, management of contaminated substances and decontamination that leads to the generation of contaminated substances are restricted; further, strict management is required, which interrupts environmental remediation.

From the perspective of optimization principle, appropriate target values for waste management should be applied to various situations to achieve realistic progress in reducing the exposure. In an existing exposure situation with elevated environmental radiation level, the target values for waste management as the source related reference levels should be selected in the range of the dose band of 1-20 mSv per year indicated in the ICRP 2007 Recommendations ${ }^{1)}$, on condition that related information are adequately shared among the stakeholders. Practical and steady environmental remediation would be possible through continuous effort in selection of reference levels; a few $\mathrm{mSv}$ to few tens of $\mathrm{mSv}$ per year during the early phases and stepwise reductions of the reference level in phases as the environmental radiation level decreases ${ }^{9}$. 


\section{Conclusions}

Protection against radiation after a nuclear accident is beyond normality and affects the economics, government, environment, society, and public psychology. To ensure smooth implementation of the optimization of protection against radiation in the existing exposure situations, it is important to further understand the reference levels, consider a long-term coexistence with the manageable radiation sources in the environment, and reduce the exposure in phases. It is easier said than done; however, we hope that this study will help the reader to understand and contribute to restore the contaminated areas.

\section{References}

1) International Commission on Radiological Protection. The 2007 Recommendations of the International Commission on Radiological Protection. ICRP Publication 103. Ann. ICRP. 2007; 37: 1-332.

2) International Commission on Radiological Protection. 1990 Recommendations of the International Commission on Radiological Protection. ICRP Publication 60. Ann. ICRP. 1991; 21: 1-83.

3) International Commission on Radiological Protection. Application of the Commission's Recommendations to the Protection of People Living in Long-term Contaminated Areas after a Nuclear Accident or a Radiation Emergency. ICRP Publication 111. Ann. ICRP. 2009; 39: 1-67.

4) Ogino H, Ichiji T, Hattori T. Verification of screening for decontamination implemented after Fukushima nuclear accident. Radiat. Prot. Dosim. 2012 August; 151: 36-42.

5) Hamada N, Ogino H. Food safety regulations: what we learned from the Fukushima nuclear accident. J. Environ. Radioact. 2012 September; 111: 83-99.

6) Hamada N, Ogino H. Problems and improvement proposals for food safety regulations during the Fukushima Daiichi Nuclear Disaster. Report from Central Research Institute of Electric Power Industry. 2011 December; L11001: 1-28. [in Japanese]

7) Hamada N, Ogino H, Fujimichi Y. Safety regulations of food and water implemented in the first year following the Fukushima nuclear accident. J. Radiat. Res. 2012 September; 53: 641-671.

8) Nuclear Safety Commission, Japan. Basic Guides for Safety Review of Category 2 Radioactive Waste Disposal. 2010 August; NSCRG: F-RW-I.02. [in Japanese]

9) Sugiyama D, Hattori T. Radiological protection from radioactive waste management in existing exposure situations resulting from a nuclear accident. Radiat. Prot. Dosim. 2013 January; 153: 74-79. 